\title{
Generating DEM of Very Narrow Baseline Stereo Using Multispectral Images
}

\author{
Yusheng Xu, Zhen Ye, Lingyun Li, Shijie Liu, Tianpeng Li, Xiaohua Tong \\ Department of Surveying and Geo-informatics \\ Tongji University \\ Shanghai, China \\ e-mail: xys198948@sina.com
}

\begin{abstract}
Traditional digital elevation model (DEM) is extracted from a pair of stereo images with a wide baseline. But wide baselines may increase the likelihood of occlusion problems and the difficulties of image matching. In this work, a method that generates DEM from multispectral images with very narrow baseline is demonstrated. A dense match algorithm based on 2-D fitting Phase Correlation (PC) method is applied to accurate estimate disparity maps of multispectral images. The experimental results using ASTER imagery show that DEM generated from multispectral images is feasible and the image pair is much easier for matching. The image matching accuracy is higher than 1/10th pixel in mountainous areas after error points eliminated. Comparing with the DEM of conventional baseline, the DEM from multispectral images has an acceptable accuracy. DEM generated from multispectral images is inferior to traditional DEM in details of mountain ranges. But the trends of the mountains and topographical features are presented clearly. And error analysis of this algorithm implies that the matching accuracy, distortion of sensors and satellite jitter could be the main factor which influence the quality of DEM extracted from multispectral images.
\end{abstract}

Index Terms-DEM generation, multispectral image, very narrow baseline stereo, phase correlation, error analysis.

\section{INTRODUCTION}

Conventional digital elevation models (DEM) are generated from one or more pairs of stereo images, which are obtained by satellites or aircrafts with a wide photography baseline. Typically, the $B / H$ ratio of the stereo pair ranges from $0.6-1$ [1], which will offer a high elevation estimation accuracy. However, a wide baseline with large viewing angles will increase the likelihood of taller objects obscuring (occluding) nearby smaller features [2] and will lead to more changes between image pairs, hence more difficulties in image matching process [3]. To those problems, narrow baseline stereovision is one of the solutions [4].

For most of multispectral sensors on board the satellite, the baselines between sensors of different bands are much smaller than traditional stereovision baseline. The images obtained from multiband sensors can avoid the occlusion problem and easier for matching process .But with bitty baseline, the stereovision of multispectral images requires highly accurate sub-pixel disparity measurement. Benefited from the development of sub-pixel phase correlation algorithm, which enables estimation of image shift with an accuracy higher than 1/20-pixel [5], narrow baseline stereovision is feasible.

In this work we demonstrate a DEM generation algorithm, of which the stereovision is composed of multispectral images with a very narrow baseline and attempt to analyze the error source for the DEM generated from multispectral images.

\section{METHOD}

The very narrow baseline stereovision principle are similar to the traditional stereovision, hence we put emphasis on the accurate measurement of disparity map of the multispectral image pairs.

The successful identification of corresponding points and accurate measurement of the disparity between them are the key technical steps for narrow baseline stereo image-matching [1]. So in this work we use the dense matching algorithm to get the corresponding points in multispectral images, and then apply the 2-D fitting PC method to measure the sub-pixel disparities of the corresponding points. Moreover, error match points are masked off and major gaps are filled in order to get accurate disparity map.

\section{A. Very Narrow Baseline Stereo Principle For Multispectral Image}

The principle of very narrow baseline stereovision use multispectral images can be expressed as follow [1] [3].

The relation between the image disparity and the ground elevation is expressed by Eq. 1

$$
d=\frac{d \cdot \sigma+B}{H \cdot \sigma} \cdot h
$$

$$
\text { Where } \begin{aligned}
H & =\text { altitude of satellite } \\
\sigma & =\text { ground resolution of image } \\
B & =\text { baseline distance of multispectral images } \\
h & =\text { ground elevation }
\end{aligned}
$$

For satellite images, we can assume that $H \gg h$ [4], and its baseline distance is determined by sensor's exposure and sample interval, then the Eq. 1 can be written as 


\section{EXPERIMENTS AND ANALYSIS}

$$
h=\frac{H \cdot \Delta t}{\Delta T} \cdot d
$$

Where $\Delta T=$ exposure interval of multispectral image pairs $\Delta t=$ line sampling interval in each image

From Eq. 2, for the multispectral images, it can be seen that the height $h$ can be directly calculated by $H, \Delta T, \Delta t$ and $d$. And on the other hand, the precious of $h$ is determined by $d$ for the fact that $H, \Delta T, \Delta t$ are constant values.

\section{B. Dense Matching Algorithm}

Search and identify the corresponding points is a main stage for stereovision. For multispectral image pairs, a pixel by pixel dense matching algorithm had been applied to identify corresponding points. Similar to the algorithm used for small baseline stereovision [6], the dense matching algorithm for multispectral image pair can be described as follow steps.

a) Image frame co-registration: Since the multispectral image pair has a slightly shift between each other, a coregistration step is applied to make a registration of the image frame.

b) Disparity estimation use 2-D fitting PC: Once the image frame co-registration is completed, a small size corresponding window based on 2-D fitting PC method is applied to scan the images. With a pixel by pixel interval, disparity in along-track direction of the pixel in the center of each window is calculated. During the scan process, the points with low correlation window had been masked off.

c) Match point processing: After the disparity estimation, the error point and masked point are removed and filled with median filter.

\section{2-D fitting Phase Correlation}

In this work a 2-D fitting PC method based on Peak Evaluation Formula (PEF) [5] is used to estimate the disparity between corresponding windows accurately. The precious of estimation can reach 1/10 1/100-pixel and the PEF method is much faster than the traditional iterative PC method [5] [7].

\section{Match Point Processing}

Some of the featureless areas may lead to the fail of phase correlation [6]. The difference between spectrums of multispectral images may also lead to significant spectral different areas that will cause error corresponding points. Therefore, median filter is also carried out to remove the false matches and fill the gaps. The median filter size that used is 7 $\times 7 \sim 11 \times 11$ pixel.

\section{A. Data And Result}

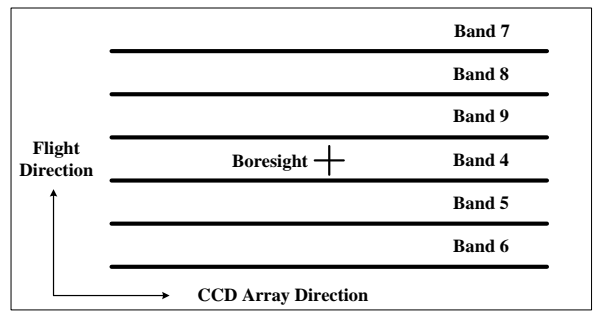

Fig. 1. Configuration of ASTER SWIR's focal plan

ASTER sensor is an imaging camera on board the Terra satellite, which was launched in December 1999. The SWIR radiometer of ASTER has six CCD linear-array sensors for the narrow bands in the shortwave infrared region [7]. All of the SWIR sensors are placed in parallel on focal plan. The exposure interval between adjacent SWIR sensors is $356.238 \mathrm{~ms}$ and the sampling interval of each line of SWIR sensors is $4.398 \mathrm{~ms}$. Figure 1 shows the configuration of SWIR sensors [8].

\section{a) ASTER Multispectral Data}

In this work, Band 6 and Band 7 images of ASTER SWIR are used as experiments data. The linear CCD array of Band 6 and Band 7 are arranged at the ends of the focal plan separately as shown in Fig. 1. That makes Band6 and Band 7 have a largest baseline than other sensor pairs. For the sensors of Band 6 and Band 7 , the $B / H$ ratio is $(4 \times 356.238 / 4.398) / 705 \mathrm{~km} \approx$ 0.0137 , and the ground resolution of the image is $30 \mathrm{~m}$. The image area is Mt. Fuji in Japan and image was obtained at 2002.10.4.

\section{b) Generate DEM}

The corresponding window of $2-\mathrm{D}$ fitting $\mathrm{PC}$ is $50 \times 50$ pixel and the median filter window is $7 \times 7$ pixel. Figure 2 shows the relative DEM based on the disparity map.
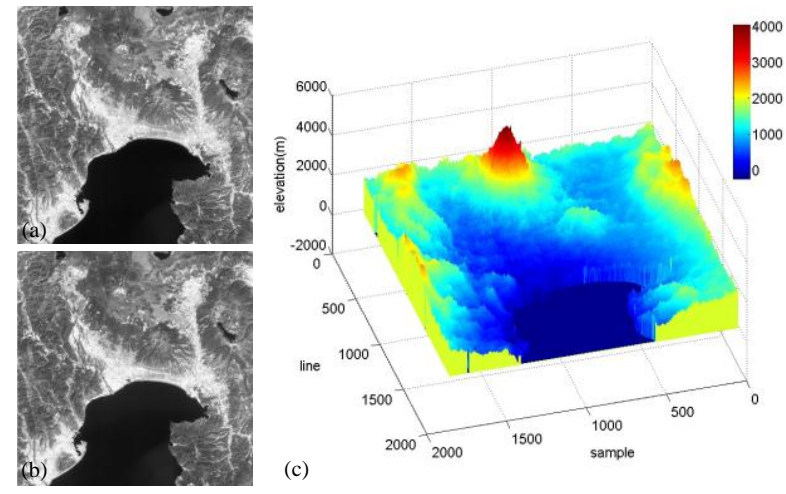

Fig. 2. (a) and (b) The image pair of Band6 and Band7. (c) Relative DEM of Band6 and Band7, 3D color view. 

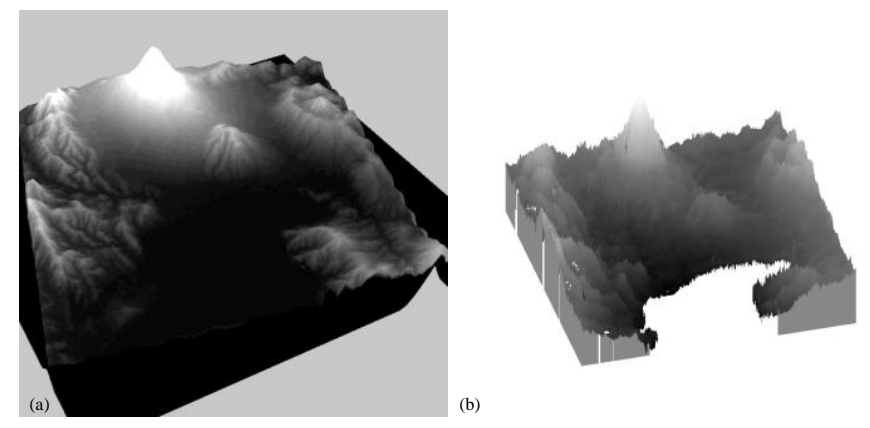

Fig. 3. (a) DEM from traditional image pairs using VNIR Band3N/Band3B and (b) DEM from multispectral images using SWIR Band6/Band7.

\section{B. Compare With The Traditional Wide Baseline DEM}

As a comparison, a traditional DEM extracted from Band $3 \mathrm{~N}$ and Band $3 \mathrm{~B}$ with a conventional $B / H$ ratio 0.6 is generated, which is shown in Fig.3. As can be seen from the figure, the DEM generated from multispectral images is inferior to traditional DEM in details of mountain ranges. But the trends of the mountains and topographical features are presented clearly. Note that, the ground resolution of $\operatorname{SWIR}(30 \mathrm{~m})$ is much lower than $\operatorname{VNIR}(15 \mathrm{~m})$, that should be taken into consideration when compare the DEMs.

A section curve has been extracted that used to make a comparison. It is obvious that, there exist elevation error in mountain's peak location and the mountain slope. But the trends of topography and relative gradient of the relative DEMs are almost the same. The "burrs" appear in section curve may be caused by the attitude pitch jitter, and this will be discussed in the following section.

\section{Error Analysis of The DEM}

The error of DEM generated from multispectral images depends on several factors, which matching accuracy, distortion of sensors and attitude jitter are the main error source.

\section{a) Effect of Matching Accuracy}

Matching accuracy is crucial to the measurement of disparity, the matching error relates to the DEM height can be expressed by Eq. 3. According to the matching accuracy of 2-D fitting PC [5], the error of DEM height caused by point matching is at least. But in fact, the matching accuracy will be limited by the viewing angle, texture of image area, size of corresponding window and so on. The relation between the height estimation error $\Delta h_{m}$ and the matching error $\Delta m$ can be expressed as

$$
\Delta h_{m}=\frac{H \cdot \Delta t}{\Delta T} \cdot \Delta m
$$

\section{b) Effect of Multispectral Sensor's Distortion}

For the disparity estimation, the distortion of multispectral sensors is also a significant error source. The disparity measurement of each sensor's element $j$ contains sensor's relative distortion $d(j)$ and real disparity disparity. The relative distortion between sensors is a constant value for each line but different for each CCD array element. Their relationship can be described by Eq. 4 and Eq. 5, where $h(j)$ and $\Delta h_{d}(j)$ represent the height estimation and its error at element $j$ separately.

$$
\begin{gathered}
h(j)=(\text { disparity }+d(j)) \cdot \frac{H \cdot \sigma}{B} \\
\Delta h_{d}(j)=d(j) \cdot \frac{H \cdot \sigma}{B}
\end{gathered}
$$

\section{c) Effect of Satellite Attitude Jitter}

It is well known that ASTER of Terra satellite suffers from an attitude jitter with a certain frequency [9] [10]. The pitch and roll components of attitude jitter appears on the disparity maps in the form of periodic patterns [7]. Since pitch jitter component which leads to the disparity in along-track direction cause the estimation error of height $\Delta h_{j}(i)$ directly, the relation between height errors and pitch jitter angles can be expressed by Eq. 6 .

$$
\Delta h_{j}(i)=\frac{H \cdot \Delta t}{\Delta T} \cdot j(i)
$$

Considering the CCD sensors is a rigid body structure, each CCD element of the sensor will be affected by jitter similarly. That means that each line of the DEM will have the same error caused by pitch jitter, if the jitter angle is large enough, the DEM will have period gutters in cross-track direction [11] [12].

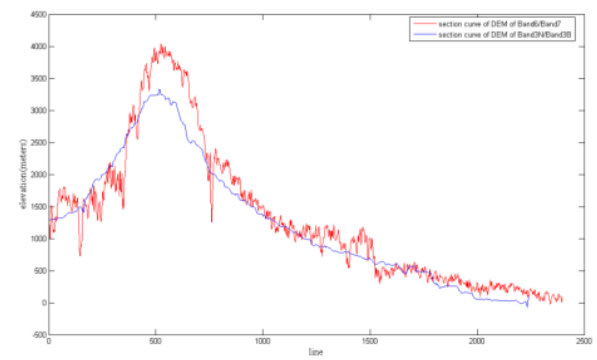

Fig. 4. Section curves of DEM from multispectral images using SWIR Band6/Band7(red) and) Band3N/Band3B (blue).

\section{CONCLUSION AND FUTURE WORKS}

Using multispectral images with a very narrow baseline to generate DEM is a new approach that previously might not have been considered suitable for stereo applications. In this paper, we demonstrated a DEM generation algorithm using multispectral images with a very low $B / H$ ratio and obtain acceptable results. The results indicate that matching accuracy, sensor's distortion and satellite jitter are main error factors of the DEM from multispectral images. Due to those factors above, the elevation accuracy and topography details of DEM 
from multispectral images are slightly inferior to traditional DEM with a wide baseline, but in urban and highly dissected mountainous areas the narrow baseline stereovision can avoid occlusion problems [1] and easier for accurate matching. In the future, we aim to increase the estimation accuracy of this algorithm and apply the improved algorithm to multispectral image pairs obtained from high resolution satellite e.g.ZY-3, SPOT5, etc. which can offer a higher ground resolution, a more stable satellite platform and multispectral camera with low distortion.

\section{ACKNOWLEDGMENT}

The work described in this paper was substantially supported by the National Natural Science Foundation of China (Project No. 41171352), National High-tech Research and Development Program (Project No. 2012AA120905 and 2012AA121302), National key Basic Research Program of China (973 Program) (Project No. 2012CB957701), Shanghai Outstanding Academic Leaders Program (Project No. 12XD1404900), and Kwang-Hua Fund for College of Civil Engineering, Tongji University. The authors would like to acknowledge Dr. A. Iwasaki at University of Tokyo for providing the ASTER data. The authors also would like to thank Dr. J. G. Liu's team and their preeminent work.

\section{REFERENCES}

[1] G. Llewellyn, K. Morgan and J. G. Liu, "Precise Subpixel Disparity Measurement From Very Narrow Baseline Stereo, " IEEE Trans. Geosci. Remote Sens.vol. 48, NO. 9, SEPTEMBER 2010J. Clerk Maxwell, A Treatise on Electricity and Magnetism, 3rd ed., vol. 2. Oxford: Clarendon, 1892, pp.68-73.

[2] J. G. Liu, G. L. K. Morgan and H. Yan, "Sub-pixel stereomatching for dem generation from narrow baseline stereo imagery," in Geoscience and Remote Sensing Symposium.
IGARSS, IEEE, 2008, vol. III, pp. 1284-1287. K. Elissa, “Title of paper if known," unpublished.

[3] J. Delon and B. Rougé, "Small baseline stereovision," J. Math Imag. Vis.,vol. 28, no. 3, pp. 209-223, Jul. 2007.

[4] T.Arai and A.Iwasaki, "Fine image matching for narrow baseline stereovision, " in Geoscience and Remote Sensing Symposium. IGARSS, IEEE, 2012, pp. 2336 - 2339.

[5] S. Nagashima, T. Aoki, T. Higuchi and K. Kobayasih, "A Subpixel Image Matching Technique Using Phase-Only Correlation," ISPACS2006, pp. 701-704, 2006.

[6] J. G. Liu and H. Yan "Phase correlation pixel-to-pixel image co-registration based on optical flow and median shift propagation", Int. J. Remote Sens., vol. 29, no. 20, pp.5943 5956, 2008.

[7] A. Iwasaki and H. Fujisada, "ASTER geometric performance, " IEEE Trans. Geosci. Remote Sens., vol. 43, no. 12, pp. 27002706, Dec. 2005.

[8] Earth Remote Sensing Data Analysis Center, ASTER User's Guide. http://www.science.aster.ersdac.or.jp/en/documnts/usersguide/part1/pdf/ Part1-4E.pdf, 2005.

[9] Y. Teshima and A. Iwasaki "Correction of attitude fluctuation of Terra spacecraft using ASTER/SWIR imagery with parallax observation", IEEE Trans. Geosci. Remote Sens., vol. 46, no. 1, pp.222 -227, 2008.

[10] A. Iwasaki, Detection and estimation satellite attitude jitter using remote sensing imagery, "Advances in Spacecraft Technologies," pp.257-272, 2011.

[11] S. Mattson, M. Robinson, A. McEwen, A. Bartels, E. BowmanCisneros, R. Li, J. Lawver, T. Tran, et al. Early Assessment of Spacecraft Jitter in LROC-NAC. 41st Lunar and Planetary Science Conference, \#1871:2010.

[12] S. Mattson, A. Bartels, A. Boyd, P. Calhoun, O. Hsu, A. McEwen, et al. Continuing analysis of spacecraft jitter in LROC-NAC. 42nd Lunar and Planetary Science Conference, \#2756:2011 